ECCOMAS Proceedia Computational Methods in Structural Dynamics and Earthquake Engineering M. Papadrakakis, M. Fragiadakis (eds.) Streamed from Athens, Greece, 28 - 30 June 2021

\title{
A COMPARATIVE STUDY ON THE ACCURACY AND EFFICIENCY OF TWO BIAXIAL HYSTERETIC MODELS SUITABLE FOR ELASTOMERIC BEARINGS
}

\author{
Nagavinothini Ravichandran ${ }^{1}$ \\ ${ }^{1}$ Department of Structures for Engineering and Architecture, University of Naples Federico II, Via \\ Claudio 21, 80125, Napoli, Italy \\ e-mail: nagavinothini.ravichandran@unina.it
}

\begin{abstract}
In common practice, uncoupled hysteretic models, that is, models that do not consider the biaxial interaction between the restoring forces along two generic orthogonal transverse directions, are typically employed to perform nonlinear time history analyses of base isolated structures. However, such models do not suitably reproduce the exact response of the structure when the system is subjected to external excitation in two orthogonal directions. Thus, the selection of suitable phenomenological model and the time integration method is important while carrying out nonlinear time history analysis (NLTHA) of base isolated structures. In the present study, a detailed investigation is carried out to assess the effectiveness of two hysteretic models to reproduce the hysteretic behaviour of the elastomeric bearings. A three-dimensional base-isolated reinforced concrete building is analysed under earthquake excitations, by employing a novel biaxial phenomenological model denominated Multiple Springs Exponential Model (MSEM) and Biaxial Bouc-Wen model (BBWM). Numerical accuracy and the computational efficiency of these methods are assessed through NLTHA. The results show that the novel method is advantageous and computationally efficient in comparison with $B B W M$.
\end{abstract}

Keywords: Hysteresis model, base isolated structure, phenomenological model, multiple springs, nonlinear time history analysis. 


\section{INTRODUCTION}

Base isolation is one of the effective response control technologies that helps in reducing the seismic response of structures by introducing isolation bearings between the foundation and superstructure of the building $[1,2,3,4]$. The continuous development in the base isolation technology and innovative isolation devices $[5,6,7,8]$ lead to a significant increase in the base isolated structures in the recent years. The behaviour of base isolated structures is highly influenced by the base isolation system, which need to be carefully investigated $[9,10,11]$. Due to the difficulties associated with the development of detailed finite element models of isolation bearings, several studies were carried out to identify a hysteresis model to reproduce the most realistic behaviour of the bearings [12].

In addition, the isolation bearings display a coupled behaviour when subjected to external excitation in two orthogonal directions [13]. Several phenomenological models are widely used to reproduce the biaxial rate-independent behaviour of seismic isolation bearings obtained from cyclic experimental tests. Biaxial differential models are mostly employed to reproduce the hysteresis phenomena, due to its accuracy and requirement of a smaller number of model parameters [14]. However, the computationally efficiency of such models are affected due to the physical insignificance of the model parameters. Also, they demand a numerical solution of a differential vector equation to obtain the response of the building at each time step of a Nonlinear Time History Analysis (NLTHA).

One of the most commonly used models is the Bouc-Wen model (BWM), but the nonlinear time history analysis of a structural model using BWM demands increased computational effort. Further, the interpretation of BWM parameters is not straightforward due to its peculiar formulation [15]. The recently formulated Biaxial Bouc-Wen model (BBWM) is capable of reproducing the biaxial behaviour of the isolation bearings. Despite its reported accuracy, the physical interpretation of model parameters and the computational time remains as a major difficulty during the response analyses of base isolated buildings.

Considering the assessment of base-isolated building response, the accuracy of the nonlinear analyses depends on the selection of suitable phenomenological model and the time integration method to solve the nonlinear equilibrium equations. In this context, the present study aims at investigating the novel coupled phenomenological model developed by Vaiana et al. [14] to simulate the rate-independent hysteretic behaviour of isolation bearings of a base isolated structure. Multiple Springs Exponential Model (MSEM), formulated from the general class of novel phenomenological model, is considered for the study. The obtained results are compared with the BBWM results in order to check the accuracy and computational efficiency of the MSEM. Further, Nonlinear Time History Analyses (NLTHAs) of a two-story baseisolated reinforced concrete building is also carried out using MSEM and BBWM to model the behaviour of isolation bearings. Considering the importance of time integration method in the NLTHA of base-isolated buildings, the widely used Newmark's constant acceleration method is used along with the BBWM model. In case of base isolated building with MSEM mode, Chang's explicit method is used for NLTHA due to reduced computational effort and the results obtained from this novel methodology is compared with the conventional procedure.

\section{HYSTERESIS MODELS}

The response of a base-isolated structure is highly influenced by the dynamic properties of the isolation devices. Hence, the selection of a suitable hysteresis model plays a major role in the nonlinear analysis of such structures. Hysteresis models, ranging from simple bilinear models to complex models and developed for particular applications, are available in the liter- 
ature $[16,17]$. In the present study, a novel biaxial phenomenological model, denominated Multiple Springs Exponential Model (MSEM) and formulated by Vaiana et al. [14], is considered along with the celebrated BBWM. The hysteresis models used to represent the behaviour of isolation bearings are presented in the following subsections.

\section{$2.1 \quad$ Bouc-Wen model}

BWM is governed by a first-order nonlinear Ordinary Differential Equation (ODE) characterized by six parameters that govern the shape and size of the hysteresis loops. The model is based in the Duhem hysteresis operator and simulates a smooth transition between elastic and post-elastic states [18,19]. Park et al. [20] extended the uniaxial model to simulate the biaxial rate-independent hysteretic behaviour of systems and materials. Finally, the generalized biaxial formulation of the model is proposed by Harvey and Gavin [21], and the components of the generalized force vector are given by:

$$
\left\{\begin{array}{l}
f_{x}\left(u_{x}\right) \\
f_{y}\left(u_{y}\right)
\end{array}\right\}=a k\left\{\begin{array}{l}
u_{x} \\
u_{y}
\end{array}\right\}+(1-a) k d\left\{\begin{array}{l}
z_{x} \\
z_{y}
\end{array}\right\}
$$

where $k$ and $d$ are parameters with dimensions of stiffness and displacement, respectively, $u_{x}$ and $u_{y}$ are the generalized displacements along $\mathrm{X}$-axis and $\mathrm{Y}$-axis, respectively, $a$ is a dimensionless parameter, and $z_{x}$ and $z_{y}$ are dimensionless variables which can be obtained from the following system of two coupled first-order nonlinear ODEs:

$$
\begin{aligned}
& \dot{z}_{x}=d^{-1}\left[A \dot{u}_{x}-z_{x}\left(b\left|\dot{u}_{x} z_{x}\right|+c \dot{u}_{x} z_{x}+b\left|\dot{u}_{y} z_{y}\right|+c \dot{u}_{y} z_{y}\right)\left(z_{x}{ }^{2}+z_{y}{ }^{2}\right)^{\frac{e-2}{2}}\right] \\
& \dot{z}_{y}=d^{-1}\left[A \dot{u}_{y}-z_{y}\left(b\left|\dot{u}_{x} z_{x}\right|+c \dot{u}_{x} z_{x}+b\left|\dot{u}_{y} z_{y}\right|+c \dot{u}_{y} z_{y}\right)\left(z_{x}{ }^{2}+z_{y}{ }^{2}\right)^{\frac{e-2}{2}}\right]
\end{aligned}
$$

where $A, b$, and $c$ are dimensionless parameters that control the shape of the hysteresis loops. Thus, BBWM requires the calibration of seven parameters to simulate the exact hysteresis behaviour of isolation bearings through experiments. BWM is used along with Newmark's constant average acceleration method to solve the nonlinear equilibrium equations. This method is unconditionally stable and also allows the use of large time steps while fulfilling the requirement for accuracy $[22,23]$. However, the computational efficiency of the method is highly affected by the convergence issues due to the conjunction of the method along with the iterative procedure [24].

\subsection{Multiple Springs Exponential Model}

The novel family of biaxial models proposed by Vaiana et al. [14] is capable of simulating the typical bidirectional kinematic rate-independent hysteretic behaviour displayed by mechanical systems and materials. The biaxial model is formulated by employing the multiple springs model [25] in conjunction with the uniaxial phenomenological models developed by Vaiana et al. [26,27]. The model was developed by introducing $n$ evenly spaced springs in a circular configuration around the origin of the adopted coordinate system as shown in Figure 1(a). Each spring is made up of two different springs in parallel such as the elastic spring with generalized tangent stiffness $k_{e}$, and a rate-independent hysteretic spring with generalized tangent stiffness $k_{b}$, as shown in Figure 1(b). The generalized tangent stiffness $k_{t}$ is obtained by summing $k_{e}$ and $k_{b}$. 


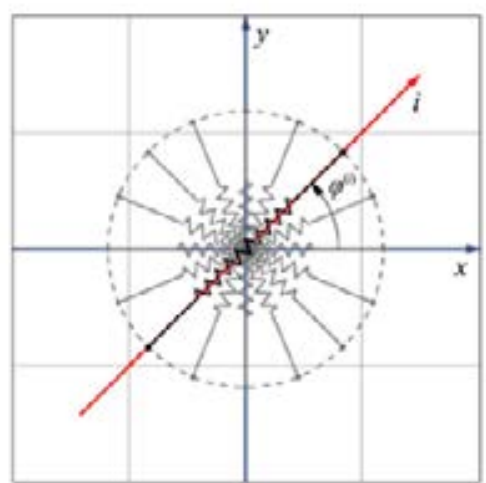

(a)

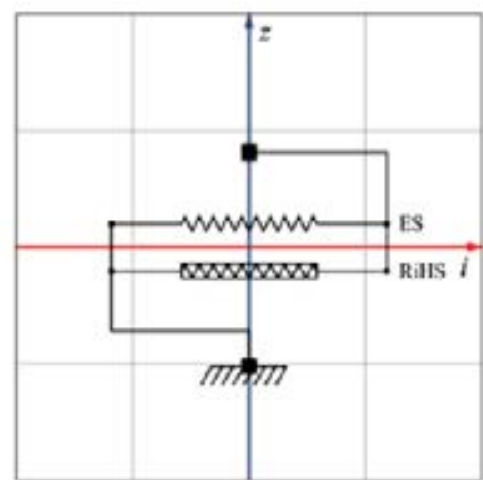

(b)

Figure 1: (a) Plan view of the multiple springs model, and (b) i-th spring [14].

The generalized force-displacement hysteresis loops characterizing the i-th spring of the proposed model may be limited to two parallel straight lines (curves), as shown in Figure 2. The integration of the general expression of the generalized tangent stiffness functions yields the expressions for the generic loading (unloading) curve and for the upper (lower) limiting curve. The integration constants are obtained by considering the intersection of generic loading (unloading) curve and upper (lower) limiting curve. The abscissa of the intersection point is represented as the generalized history variable.

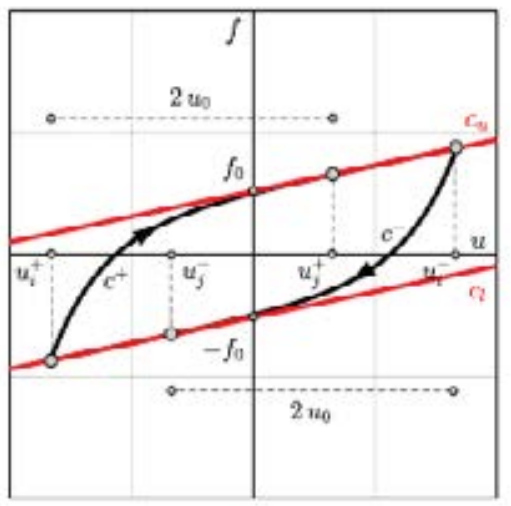

(a)

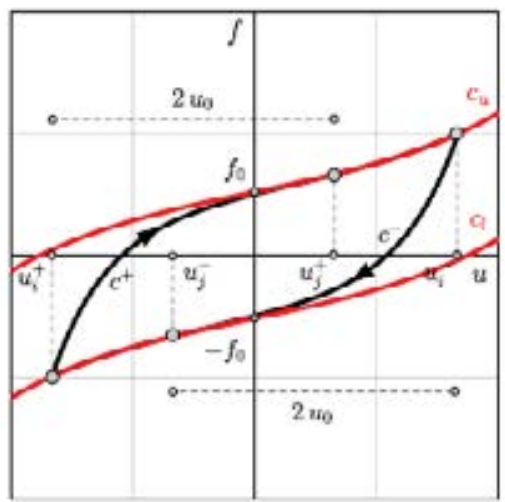

(b)

Figure 2: Hysteresis loop limited by two parallel (a) straight lines, and (b) curves [14].

Multiple Springs Exponential Model (MSEM) considered in the present study belongs to this family of biaxial models, which is obtained by selecting a suitable generalized tangent stiffness functions and then by deriving the particularized expression of the generalized force, history variable, and internal constant parameters. For simplicity, same properties are assigned for all the springs in the model, thus obtaining an isotropic model. MSEM is a more sophisticated model where the typical generalized force-displacement hysteresis loop of the ith spring is limited by two parallel straight lines or curves. This model requires four parameters to simulate the complex biaxial hysteresis phenomena. The general expression of the generalized tangent stiffness functions can be obtained from the sum of following expressions of $k_{e}$ and $k_{h}$ :

$$
k_{e}(u)=-2 \beta+\beta\left(e^{\beta u}+e^{-\beta u}\right) \text { on }(-\infty, \infty)
$$




$$
\begin{aligned}
& k_{h}\left(u, u_{j}^{+}\right)=\left\{\begin{array}{cc}
k_{b}+\left(k_{a}-k_{b}\right) e^{-\alpha\left(u-u_{j}^{+}+2 u_{0}\right)} & \text { on }\left[u_{j}^{+}-2 u_{0}, u_{j}^{+}[\right. \\
k_{b} & \text { on } \left.] u_{j}^{+}, \infty\right),
\end{array}\right. \\
& k_{h}\left(u, u_{j}^{-}\right)=\left\{\begin{array}{cc}
k_{b}+\left(k_{a}-k_{b}\right) e^{-\alpha\left(-u+u_{j}^{-}+2 u_{0}\right)} & \text { on } \left.] u_{j}^{-}, u_{j}^{-}+2 u_{0}\right] \\
k_{b} & \text { on }\left(-\infty, u_{j}^{-}[\right.
\end{array}\right.
\end{aligned}
$$

where $k_{a}, k_{b}, \alpha$, and $\beta$ are the four model parameters that fulfill the following conditions: $k_{a}>k_{b}, k_{a}>0, \alpha>0$, whereas $\beta$ is real.

The general expression of the $i$-th spring generic loading and unloading curves can be written as follows:

$$
\begin{aligned}
& c^{+}\left(u, u_{j}^{+}\right)=-2 \beta u+e^{\beta u}-e^{-\beta u}+k_{b} u-\frac{\left(k_{a}-k_{b}\right)}{\alpha}\left[e^{-\alpha\left(u-u_{j}^{+}+2 u_{0}\right)}-e^{-2 \alpha u_{o}}\right]+f_{0} \\
& c^{-}\left(u, u_{j}^{-}\right)=-2 \beta u+e^{\beta u}-e^{-\beta u}+k_{b} u+\frac{\left(k_{a}-k_{b}\right)}{\alpha}\left[e^{-\alpha\left(-u+u_{j}^{-}+2 u_{0}\right)}-e^{-2 \alpha u_{o}}\right]-f_{0}
\end{aligned}
$$

Further, the expressions of the upper and lower limiting curves are given by:

$$
\begin{aligned}
& c_{u}(u)=-2 \beta u+e^{\beta u}+e^{-\beta u}+k_{b} u+f_{0} \\
& c_{l}(u)=-2 \beta u+e^{\beta u}-e^{-\beta u}+k_{b} u-f_{0}
\end{aligned}
$$

The general equations used to evaluate the generalized history variable for the generic loading and unloading cases are as follows:

$u_{j}^{+}=u_{p}+2 u_{0}+\frac{1}{\alpha} \ln \left[+\frac{\alpha}{k_{a}-k_{b}}\left(-2 \beta u_{p}+e^{\beta u_{p}}-e^{-\beta u_{p}}+k_{b} u_{p}+\frac{\left(k_{a}-k_{b}\right)}{\alpha} e^{-2 \alpha u_{0}}+f_{0}-\right.\right.$ $\left.\left.f_{p}\right)\right]$

$u_{j}^{-}=u_{p}-2 u_{0}-\frac{1}{\alpha} \ln \left[-\frac{\alpha}{k_{a}-k_{b}}\left(-2 \beta u_{p}+e^{\beta u_{p}}-e^{-\beta u_{p}}+k_{b} u_{p}-\frac{\left(k_{a}-k_{b}\right)}{\alpha} e^{-2 \alpha u_{0}}-f_{0}-\right.\right.$ $\left.\left.f_{p}\right)\right]$

The expression of the constant parameter $u_{0}$ can be derived by assuming that the upper (lower) limiting curves and generic loading (unloading) curves have the same generalized tangent stiffness functions at their intersection point. It can be given by:

$$
u_{0}=-\frac{1}{2 \alpha} \ln \left(\frac{\delta_{k}}{k_{a}-k_{b}}\right)
$$

Further, the expression for the constant parameter $f_{0}$ is obtained as follows:

$$
f_{0}=\frac{k_{a}-k_{b}}{2 \alpha}\left(1-e^{-2 \alpha u_{0}}\right)
$$

In the present study, this novel coupled phenomenological model is used along with an explicit unconditionally stable time integration method. This strategy avoids the use of iterative procedures for solving nonlinear equilibrium equations and it is useful when complex prob- 
lems need to be addressed [28,29]. The explicit time integration method considered in the study represents a specific algorithm developed by Chang [30] and adopted by Vaiana et al. [31] for base-isolated structures. In case of non-stiffening systems, this method tends to be unconditionally stable and has a second-order accuracy. Further, the method does not suffer from numerical damping and displays only a small relative period error.

\section{NUMERICAL MODEL}

A three dimensional (3D) structural model with two substructures such as the $n$-story superstructure and a base isolation system is considered to assess the accuracy and computational efficiency of the proposed model. The 3D model defined in global, right-handed cartesian coordinate system attached to the mass centre of the base isolation system is shown in Figure 3. The nonlinear analysis of the base isolated structure is carried out considering the following assumptions: (a) the deformation of the superstructure is within the linear elastic range during the earthquake excitation, (b) the floor diaphragm is infinitely rigid in its own plane, (c) the columns are axially inextensible, (d) the beams are flexurally rigid and axially inextensible, (e) the base isolation bearings are axially inextensible.

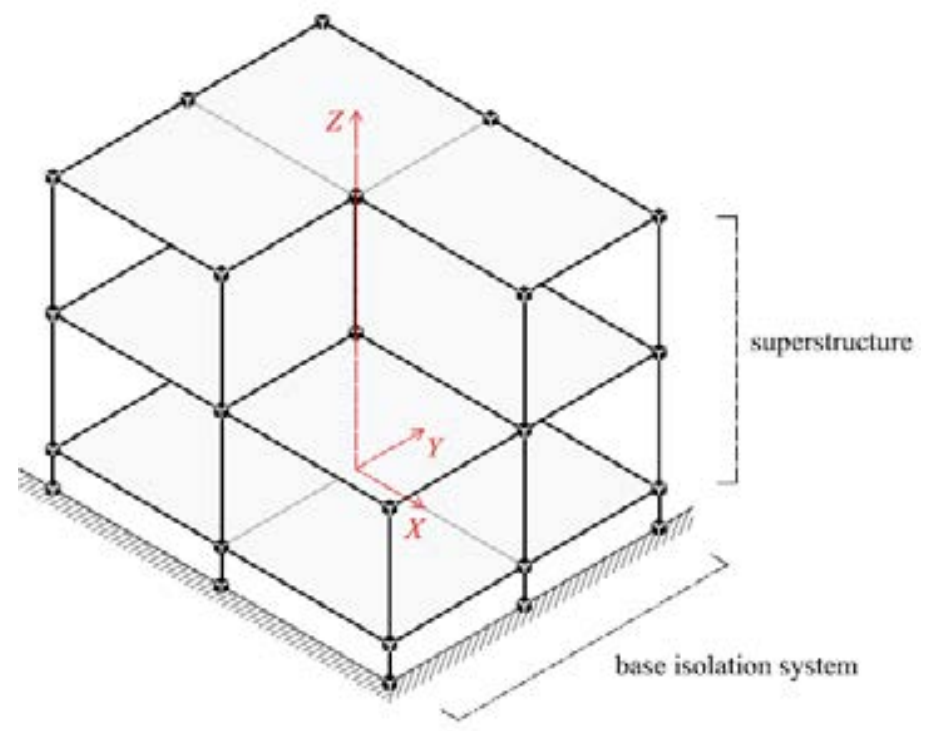

Figure 3: Three-dimensional model of base isolated structure.

The superstructure of the base isolated structure is a two-story reinforced concrete structure with vertical geometrical irregularity. The plan dimension of the building is $10.0 \mathrm{~m} \times 8.0 \mathrm{~m}$ with a story height of $3.50 \mathrm{~m}$. The weight of the superstructure is $1802.90 \mathrm{kN}$. The first three natural periods of the superstructure are $0.15 \mathrm{~s}, 0.14 \mathrm{~s}$ sand $0.10 \mathrm{~s}$. The total weight of base isolation system with 9 elastomeric bearings is $914.90 \mathrm{kN}$. The bearings are placed between the superstructure columns full diaphragm. The base isolation system is designed to provide an effective isolation period of $2.25 \mathrm{~s}$ and an effective viscous damping of $15 \%$ at the design displacement $0.50 \mathrm{~m}$. As per the assumptions considered for the nonlinear analysis of the structure, the number of degrees-of-freedom of the super structure and the isolation system are equal to 6 and 3 , respectively.

The nonlinear equilibrium equation considered for the analysis of the Multi-Degree-OfFreedom (MDOF) base isolated structure is given by:

$$
M \ddot{u}(t)+C \dot{u}(t)+K u(t)+f_{n}(t)=p(t)
$$


where, $u(t), \dot{u}(t)$, and $\ddot{u}(t)$ are the generalized displacement, velocity, acceleration vectors, respectively, $M, C$, and $K$ are the generalized constant mass, damping and elastic stiffness matrices, respectively, $f_{n}(t)$ is the generalized nonlinear force vector, and $p(t)$ is the generalized external force vector at time $t$.

For the adopted base isolated structure idealization, the motion vectors of the model relative to the ground can be written as follows:

$$
u=\left\{\begin{array}{l}
u_{b} \\
u_{s}
\end{array}\right\}, \dot{u}=\left\{\begin{array}{l}
\dot{u}_{b} \\
\dot{u}_{s}
\end{array}\right\}, \ddot{u}=\left\{\begin{array}{l}
\ddot{u}_{b} \\
\ddot{u}_{s}
\end{array}\right\}
$$

where, $u_{b}, \dot{u}_{b}, \ddot{u}_{b}\left(u_{s}, \dot{u}_{s}, \ddot{u}_{s}\right)$ are the displacement, velocity and acceleration vectors of base isolation device (superstructure) of size $3 \times 1$ ( $3 \mathrm{nx} 1)$, respectively.

The force vectors are given by:

$$
f_{n}=\left\{\begin{array}{c}
f_{n b} \\
0
\end{array}\right\}, p=-\left[\begin{array}{cc}
M_{b} & 0^{T} \\
0 & M_{s}
\end{array}\right]\left\{\begin{array}{l}
R_{b} \\
R_{s}
\end{array}\right\} \ddot{u}_{g}
$$

where, $f_{n b}$ is the nonlinear force vector of the base isolation system with dimension $3 \times 1$, $R_{b}\left(R_{s}\right)$ is the influence matrix of base isolation system (superstructure), and $\ddot{u}_{g}$ is the ground acceleration vector of dimension $3 \times 1$.

\section{HYSTERESIS MODEL PARAMETERS}

The parameters of the biaxial Bouc-Wen model adopted to simulate the behaviour of the elastomeric bearings are shown in Table 1. MSEM requires calibration of four parameters $\left(k_{a}, k_{b}, \alpha, \beta\right)$ and ' $\mathrm{n}$ ' number of springs. MSEM model parameters are identified using Particle Swarm Optimization (PSO) algorithm [32] which is a computational technique with easy implementation and convergence properties. In the algorithm, a set of random parameters are first initialized, and the value of each parameter is iteratively modified. The optimization is performed by considering the interaction among the parameters and the objective function is minimized. MSEM parameters are calibrated in the present study by minimizing the following objective function:

$$
\Theta=\sum_{j} \sqrt{\left(f_{j}^{M S E M}-f_{j}\right)^{2}} \text { with } j=x, y
$$

where, $f_{j}^{M S E M}$ is the $\mathrm{j}$-th component of the generalized force vector computed using MSEM and $f_{j}$ is the $\mathrm{j}$-th component of the generalized force vector describing the behaviour of the isolation bearings using BWM. The following PSO parameters are used to avoid premature convergence of the solution in the local optimum points:

- population size: 100 ,

- number of iterations: 200,

- acceleration factors: $\mathrm{c}_{1}=\mathrm{c}_{2}=2$,

- maximum and minimum inertia weights: $\mathrm{w}_{\max }=0.9$ and $\mathrm{w}_{\min }=0.4$.

The adopted MSEM parameters are listed in Table 2. The comparison of hysteresis loops of BWM and MSEM model with the calibrated parameters are shown in Figure 4. The plots are obtained by imposing eight-shaped displacement orbit given by $u_{x}=a_{o} \sin \omega t$ and $u_{y}=a_{o} \sin 2 \omega t$, with $a_{o}=0.5 \mathrm{~m}$ and $\omega=2 \pi \mathrm{rad} / \mathrm{s}$. It can be clearly seen that the MSEM model is capable of representing the hysteresis behaviour of the isolation bearing with reduced number of parameters, in comparison with BWM. 


\begin{tabular}{lllllll}
\hline $\mathrm{K}[\mathrm{N} / \mathrm{m}]$ & $\mathrm{A}$ & $\mathrm{a}$ & $\mathrm{b}$ & $\mathrm{c}$ & $\mathrm{d}[\mathrm{m}]$ & $\mathrm{e}$ \\
\hline $1.83 \mathrm{e} 6$ & 1 & 0.10 & 1.00 & 0.00 & 0.017 & 1.00 \\
\hline
\end{tabular}

Table 1: BWM parameters used in the NLTHAs.

\begin{tabular}{lllll}
\hline $\mathrm{n}$ & $\mathrm{k}_{\mathrm{a}}[\mathrm{N} / \mathrm{m}]$ & $\mathrm{k}_{\mathrm{b}}[\mathrm{N} / \mathrm{m}]$ & $\alpha\left[\mathrm{m}^{-1}\right]$ & $\beta[\mathrm{N} / \mathrm{m}]$ \\
\hline 16.00 & $5.78 \mathrm{e} 8$ & $1.83 \mathrm{e} 5$ & $1.04 \mathrm{e} 4$ & 0 \\
\hline
\end{tabular}

Table 2: MSEM parameters used in the NLTHAs.

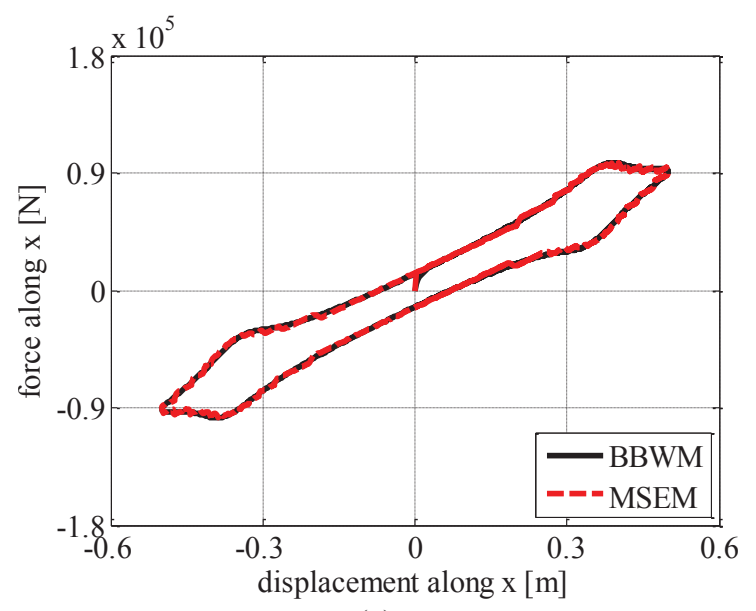

(a)

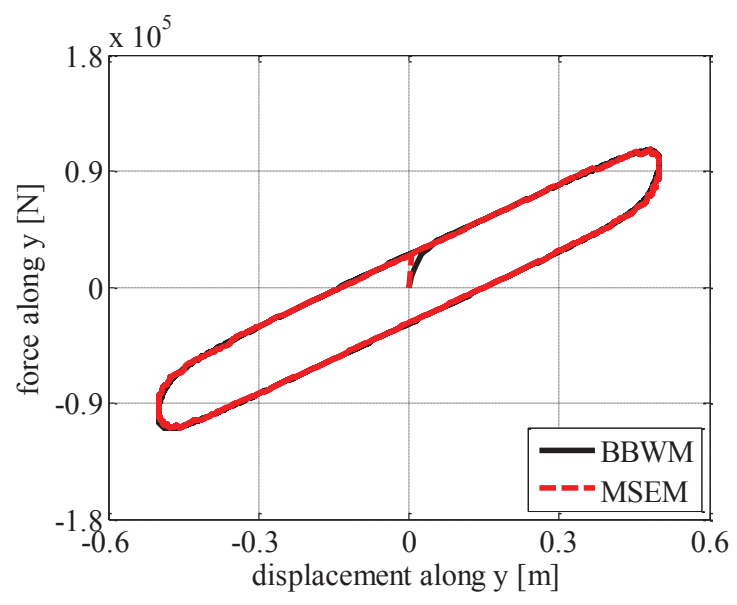

(b)

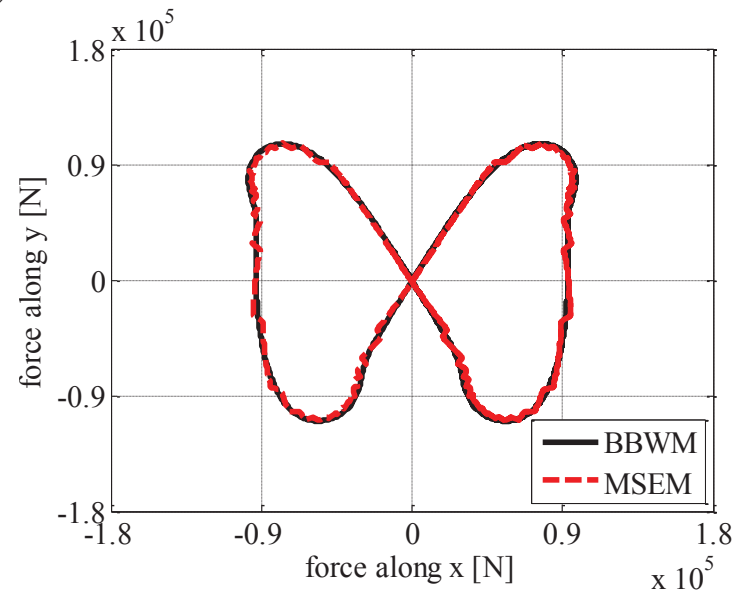

(c)

Figure 4: Comparison between BBWM and MSEM hysteresis loops along (a) X-axis, (b) Y-axis, and (c) generalized force paths by applying eight-shaped generalized displacement orbit.

The numerical model has been programmed in MATLAB and run on a computer with Intel ${ }^{\circledR}$ Core ${ }^{\mathrm{TM}}$ i5-5200U processor and a CPU at $2.20 \mathrm{GHz}$ with 8.00 GB RAM. The computational time of BBWM is $20.97 \mathrm{~s}$, whereas the MSEM model required only $0.53 \mathrm{~s}$, which is about $2.50 \%$ of the BBWM computational time. Along with the computational time, the model also exhibits acceptable accuracy with reduced number of model parameters, making the calibration process easier. 


\section{NUMERICAL ANALYSES OF A BASE-ISOLATED STRUCTURE}

The response of the base isolated structure under bidirectional earthquake excitation is presented in this section. In the analyses, the BBWM and MSEM are used to reproduce the dynamic behaviour of the isolation bearings of the base isolated structure. Further, conventional time integration method is used along with BBWM to numerically integrate the nonlinear equilibrium equations, whereas Chang's explicit method is used along with the novel MSEM. The NLTHA of the base isolated structure is performed by imposing Northridge earthquake acceleration time history along $\mathrm{X}$-axis and $\mathrm{Y}$-axis as shown in Figure 5.

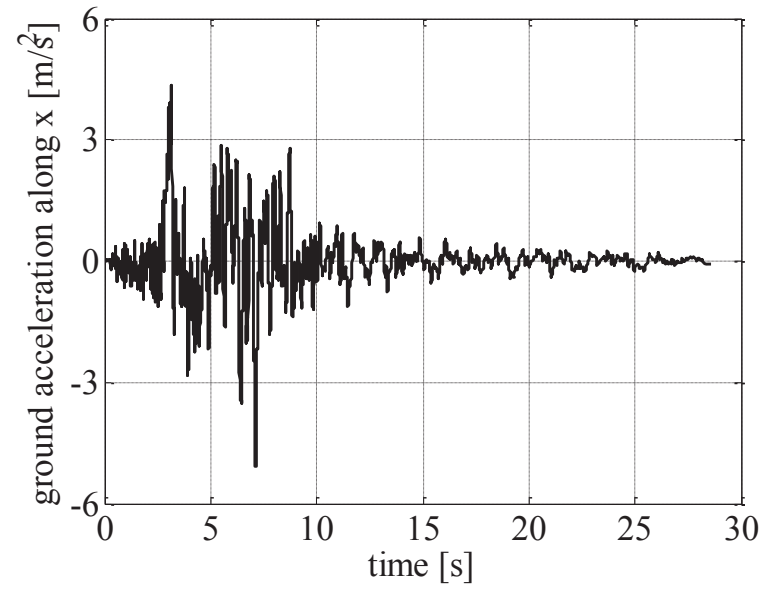

(a)

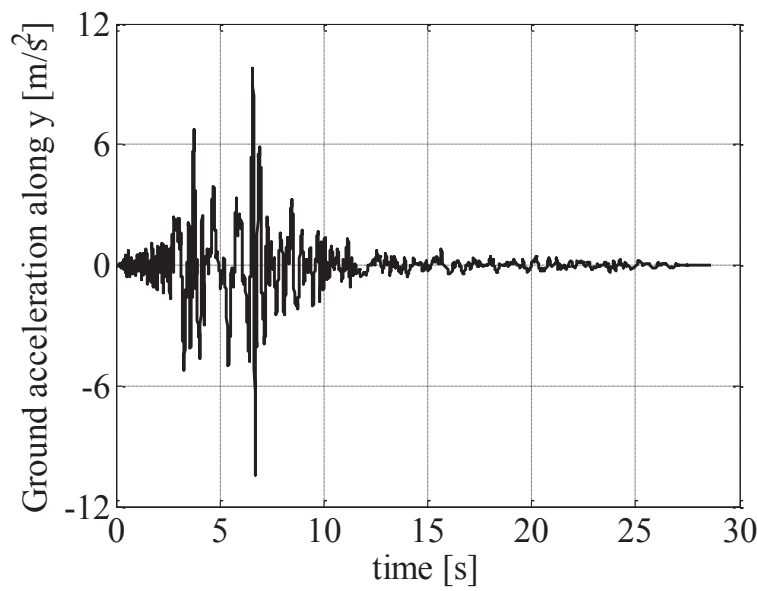

(b)

Figure 5: Ground acceleration time histories of Northridge earthquake along (a) X-axis and (b) Y-axis.

The numerical results and computational time are compared to assess the accuracy and computational efficiency of the solution strategies. The first order nonlinear ODE in the BWM is solved using the unconditionally stable semi-implicit Range-Kutta method with 50 number of steps [33]. The results of the NLTHAs are listed in Table 3. It can be clearly seen that the maximum and minimum values of the base isolation system mass centre displacements $(u)$, velocities $(\dot{u})$ and accelerations $(\ddot{u})$ obtained using BBWM and MSEM are almost equal. In addition to the seismic response of the base isolated structure, the total computational time tct required to perform NLTHAs by BBWM and MSEM are also given in Table 3. It can be clearly seen that the MSEM reduces the computational time drastically. Since, the obtained computational time highly depends on the CPU speed, relevant memory and background process running on the computer, an additional parameter tct $p$ which is the total computational time percentage is also evaluated as follows:

$$
\text { MSEM } t c t p[\%]=\frac{\text { MSEM } t c t}{\text { BBWM } t c t} \cdot 100
$$

This parameter provides a more meaningful measure of the computational benefits associated with MSEM, in comparison with BBWM. The value of the tctp in the order of $0.87 \%$ highlights the advantages of implementing this model in structural analysis tools and commercial computer programs.

Further, the comparison of displacement, velocity and acceleration response measured at the base isolation system mass centre obtained using BBWM and MSEM models are shown in Figure 6(a) to 6(f). The results clearly highlight the accuracy of the novel biaxial phenomenological model in comparison with the BBWM. A minor difference arises in terms of hyster- 
etic response of the isolation bearings in Figure 6(g) and 6(h) due to different formulations of the models to be properly calibrated on experimental tests.

\begin{tabular}{lllll}
\hline & & BBWM & MSEM & $\boldsymbol{\varepsilon}[\%]$ \\
\hline tct $[\mathrm{s}]$ & & 12104 & 105 & \\
tctp $[\%]$ & & - & 0.87 & \\
$u_{x}[\mathrm{~m}]$ & Max & 0.33 & 0.33 & 0.00 \\
& Min & -0.33 & -0.33 & 0.00 \\
$u_{y}[\mathrm{~m}]$ & Max & 0.23 & 0.25 & 8.00 \\
& $\min$ & -0.18 & -0.21 & 14.29 \\
$\dot{u}_{x}\left[\mathrm{~ms}^{-1}\right]$ & Max & 0.89 & 0.90 & 1.11 \\
& $\min$ & -0.83 & -0.82 & -1.20 \\
$\dot{u}_{y}\left[\mathrm{~ms}^{-1}\right]$ & Max & 0.98 & 1.08 & 9.26 \\
& $\min$ & -0.85 & -0.89 & 1.12 \\
$\ddot{u}_{x}\left[\mathrm{~ms}^{-2}\right]$ & Max & 5.16 & 4.90 & -5.04 \\
& $\min$ & -3.93 & -3.83 & -2.54 \\
$\ddot{u}_{y}\left[\mathrm{~ms}^{-2}\right]$ & Max & 10.37 & 10.47 & 0.96 \\
& $\min$ & -10.55 & -10.30 & -2.37 \\
\hline
\end{tabular}

Table 3: NLTHAs results under Northridge excitation.

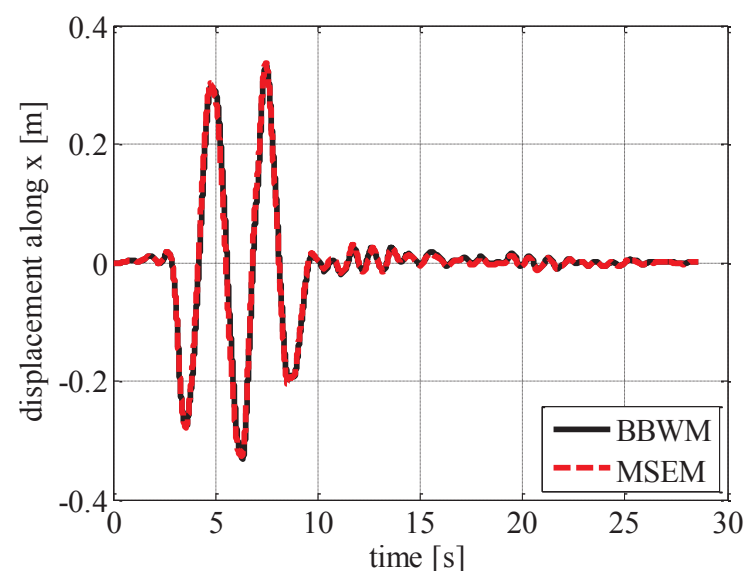

(a)

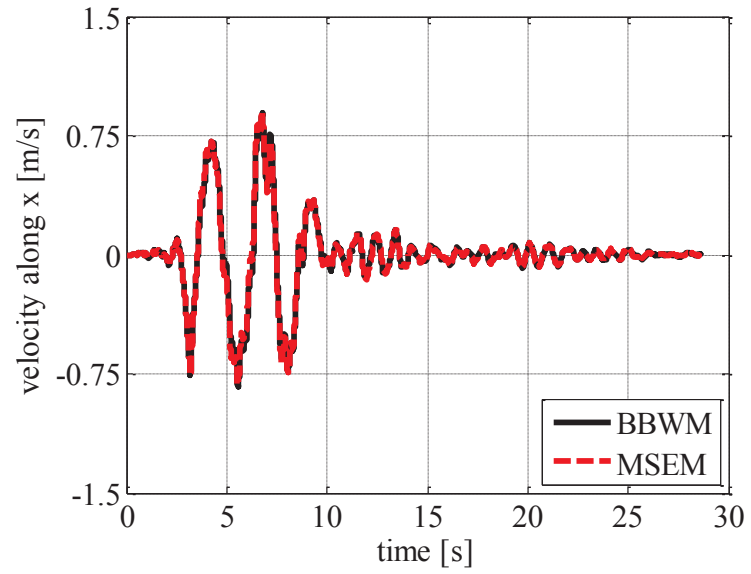

(c)

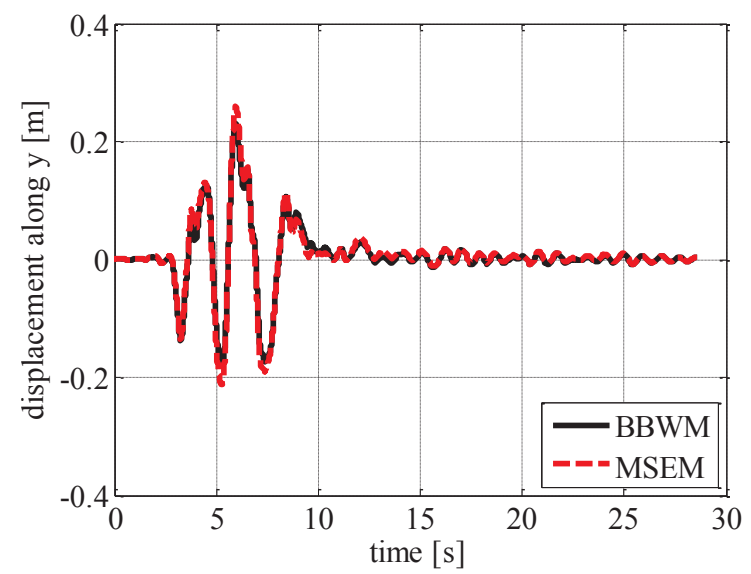

(b)

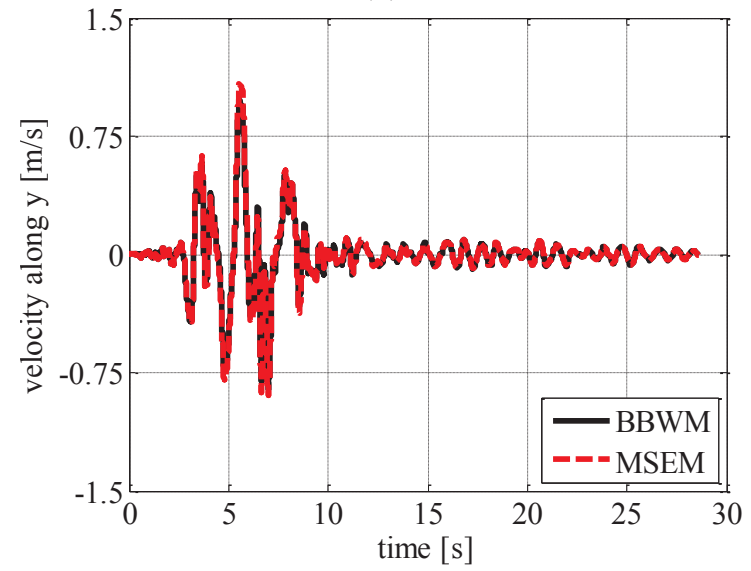

(d) 


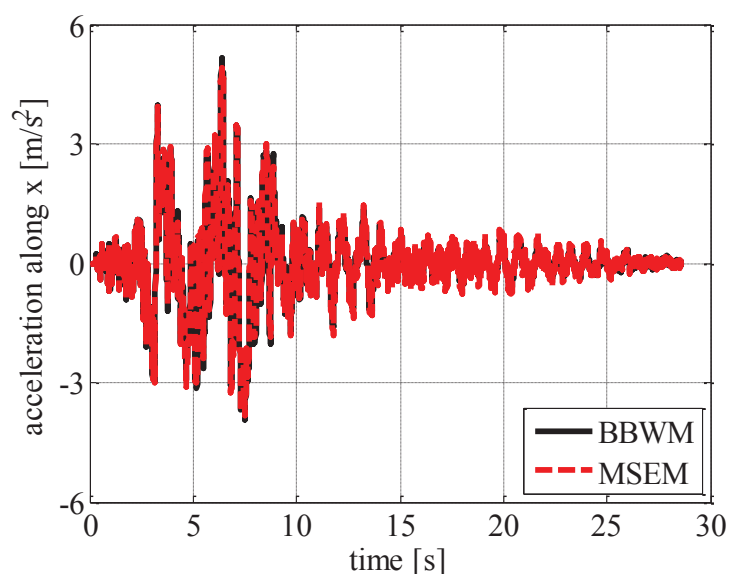

(e)

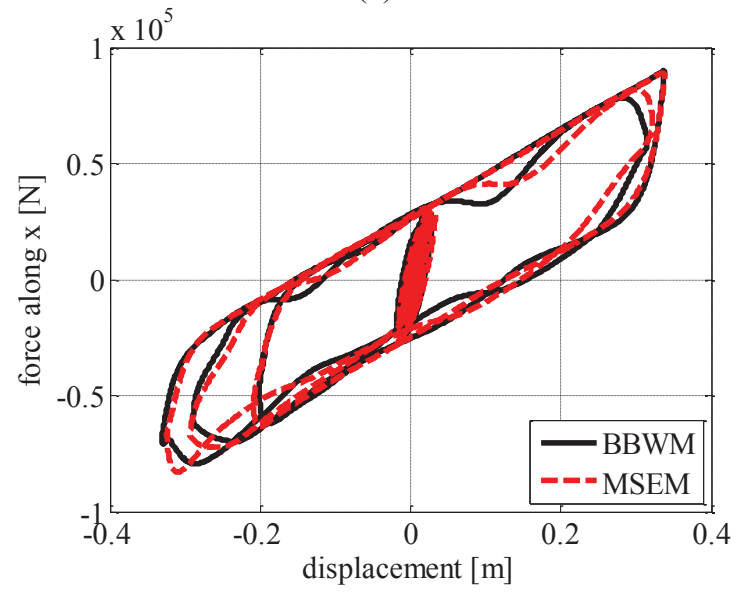

(g)

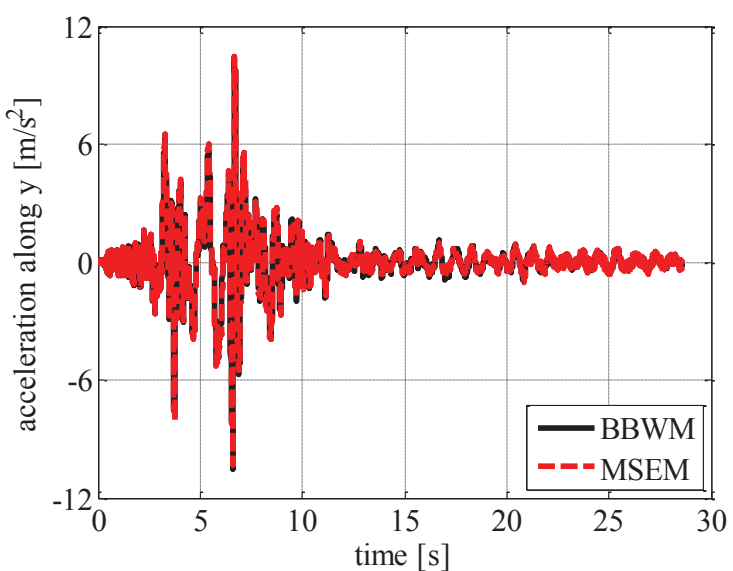

(f)

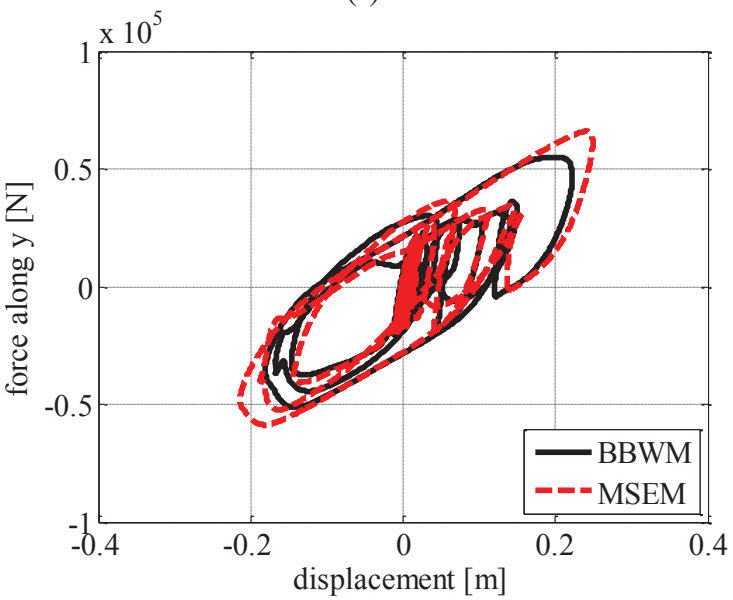

(h)

Figure 6: Seismic response of the base isolated structure (a) displacement along X-axis, (b) displacement along $\mathrm{Y}$-axis, (c) velocity along $\mathrm{X}$-axis, (d) velocity along $\mathrm{Y}$-axis, (e) acceleration along X-axis, (f) acceleration along $\mathrm{y}$-axis, (g) hysteresis loops along $\mathrm{X}$-axis, and (h) hysteresis loops along $\mathrm{Y}$-axis.

\section{CONCLUSIONS}

In the present study, the accuracy and computational efficiency of the two biaxial hysteretic models to reproduce the behaviour of elastomeric bearings is assessed. A novel biaxial phenomenological model denominated Multiple Springs Exponential Model is compared with the commonly used Biaxial Bouc-Wen Model. The novel model is found to be advantageous in comparison with BBWM due its reduced number of model parameters, physical interpretation of parameters, easier calibration, and reduced computational time.

Further, NLTHA of a three-dimensional base isolated reinforced concrete building is also carried out. Considering the importance of time integration methods employed in NLTHA of base isolated structures, Newmark's constant average acceleration method and Chang's explicit method are used along with BBWM and MSEM, respectively. The dynamic response of the base isolated structure obtained using the MSEM model and Chang's explicit method closely matches with the results from the conventional procedure using BBWM and Newmark's method. Further, the total computational time percentage of the novel method is only around $0.87 \%$ which highlights the computational efficiency and suitability of this model for implementation in structural analysis tools. 


\section{REFERENCES}

[1] D. Losanno, H.A. Hadad, G. Serino, Seismic behavior of isolated bridges with additional damping under far-field and near fault ground motion. Earthquakes and Structures, 13(2), 119-130, 2017.

[2] D. Losanno, H.A. Hadad, G. Serino, Design charts for eurocode-based design of elastomeric seismic isolation systems. Soil Dynamics and Earthquake Engineering, 119, 488-498, 2019.

[3] D. Losanno, M. Spizzuoco, A. Calabrese, Bidirectional shaking-table tests of unbonded recycled-rubber fiber-reinforced bearings (RR-FRBs). Structural Control and Health Monitoring, 26(9), e2386, 2019.

[4] N. Vaiana, R. Capuano, S. Sessa, F. Marmo, L. Rosati, Nonlinear Dynamic Analysis of Seismically Base-Isolated Structures by a Novel OpenSees Hysteretic Material Model. Applied Sciences, 11(3), 900, 2021.

[5] A. Calabrese, D. Losanno, M. Spizzuoco, S. Strano, M. Terzo, Recycled Rubber Fiber Reinforced Bearings (RR-FRBs) as base isolators for residential buildings in developing countries: The demonstration building of Pasir Badak, Indonesia. Engineering Structures, 192, 126-144, 2019.

[6] N. Vaiana, S. Sessa, M. Paradiso, L. Rosati, Accurate and efficient modeling of the hysteretic behavior of sliding bearings. In 7th International Conference on Computational Methods in Structural Dynamics and Earthquake Engineering (COMPDYN 2019), Crete, Greece, June, 2019. doi: 10.7712/120119.7304.19506

[7] D. Losanno, A. Calabrese, I.E. Madera-Sierra, M. Spizzuoco, J. Marulanda, P. Thomson, G. Serino, Recycled versus natural-rubber fiber-reinforced bearings for base isolation: review of the experimental findings. Journal of Earthquake Engineering, 1-20, 2020.

[8] D. Losanno, F. Palumbo, A. Calabrese, T. Barrasso, N. Vaiana, Preliminary Investigation of Aging Effects on Recycled Rubber Fiber Reinforced Bearings (RRFRBs). Journal of Earthquake Engineering, 1-18, 2021.

[9] D. Losanno, I.E.M. Sierra, M. Spizzuoco, J. Marulanda, P. Thomson, Experimental assessment and analytical modeling of novel fiber-reinforced isolators in unbounded configuration. Composite Structures, 212, 66-82, 2019.

[10] D. Losanno, N. Ravichandran, F. Parisi, A. Calabrese, G. Serino, Seismic performance of a Low-Cost base isolation system for unreinforced brick Masonry buildings in developing countries. Soil Dynamics and Earthquake Engineering, 141, 106501, 2021.

[11] I.E.M. Sierra, D. Losanno, S. Strano, J. Marulanda, P. Thomson, Development and experimental behavior of HDR seismic isolators for low-rise residential buildings. Engineering Structures, 183, 894-906, 2019.

[12] N. Ravichandran, A comparative study of phenomenological hysteretic models with application to recycled rubber-fibre reinforced bearings. Journal of Vibroengineering, 22(8), 1798-1813, 2020.

[13] D. Losanno, I.E.M. Sierra, M. Spizzuoco, J. Marulanda, P. Thomson, P, Experimental performance of unbonded polyester and carbon fiber reinforced elastomeric isolators under bidirectional seismic excitation. Engineering Structures, 209, 110003, 2020. 
[14] N. Vaiana, D. Losanno, N. Ravichandran, A novel family of multiple springs models suitable for biaxial rate-independent hysteretic behavior. Computers \& Structures, 244, 106403, 2021.

[15] A.E. Charalampakis, V.K. Koumousis, Identification of Bouc-Wen hysteretic systems by a hybrid evolutionary algorithm. Journal of Sound and Vibration, 314(3-5), 571-585, 2008.

[16] J. Lubliner, Plasticity theory. Courier Corporation, 2008.

[17] C.S. Tsai, T.C. Chiang, B.J. Chen, S.B. Lin, An advanced analytical model for high damping rubber bearings. Earthquake engineering \& structural dynamics, 32(9), 13731387, 2003.

[18] Y.K. Wen, Method for random vibration of hysteretic systems. Journal of the engineering mechanics division, 102(2), 249-263, 1976.

[19] Y.K. Wen, Equivalent linearization for hysteretic systems under random excitation. Journal of applied mechanics, 47(1), 150-154, 1980.

[20] Y.J. Park, Y.K. Wen, A.H.S. Ang, Random vibration of hysteretic systems under bidirectional ground motions. Earthquake engineering \& structural dynamics, 14(4), $543-$ $557,1986$.

[21] P.S. Harvey Jr, H.P. Gavin, Truly isotropic biaxial hysteresis with arbitrary knee sharpness. Earthquake engineering \& structural dynamics, 43(13), 2051-2057, 2014.

[22] K.J. Bathe, Finite element procedures. Klaus-Jurgen Bathe, 2006.

[23] F. Greco, R. Luciano, G. Serino, N. Vaiana, A mixed explicit-implicit time integration approach for nonlinear analysis of base-isolated structures. Annals of Solid and Structural Mechanics, 10(1-2), 17-29, 2018.

[24] R. Clough, J. Penzien, Dynamics of structures. McGraw-Hill, New York, 1993.

[25] A. Wada, M. Kinoshita, Elastic plastic dynamic 3-dimensional response analysis by using multiple shear spring model Part1 and Part2. In Summaries of Technical Papers of Annual Meeting (pp. 313-316). 313-316 (in Japanese) Tokai, Japan, 1985.

[26] N. Vaiana, S. Sessa, F. Marmo, L. Rosati, A class of uniaxial phenomenological models for simulating hysteretic phenomena in rate-independent mechanical systems and materials. Nonlinear Dynamics, 93(3), 1647-1669, 2018.

[27] N. Vaiana, S. Sessa, L. Rosati, A generalized class of uniaxial rate-independent models for simulating asymmetric mechanical hysteresis phenomena. Mechanical Systems and Signal Processing, 146, 106984, 2021.

[28] S. Sessa, F. Marmo, N. Vaiana, L. Rosati, A computational strategy for Eurocode 8compliant analyses of reinforced concrete structures by seismic envelopes. Journal of Earthquake Engineering, 1-34, 2018.

[29] F. Marmo, S. Sessa, N. Vaiana, D. De Gregorio, L. Rosati, Complete solutions of threedimensional problems in transversely isotropic media. Continuum Mechanics and Thermodynamics, 32(3), 775-802, 2020.

[30] S.Y. Chang, Family of structure-dependent explicit methods for structural dynamics. Journal of Engineering Mechanics, 140(6), 06014005, 2014. 
[31] N. Vaiana, S. Sessa, M. Paradiso, F. Marmo, L. Rosati, An Efficient Computational Strategy for Nonlinear Time History Analysis of Seismically Base-Isolated Structures. In Conference of the Italian Association of Theoretical and Applied Mechanics (pp. 1340-1353). Springer, Cham, September, 2019. https://doi.org/10.1007/978-3-030-41057-5_108

[32] J. Kennedy, R. Eberhart, Particle swarm optimization. In Proceedings of ICNN'95international conference on neural networks (Vol. 4, pp. 1942-1948). IEEE, November, 1995.

[33] H.H. Rosenbrock, Some general implicit processes for the numerical solution of differential equations. The Computer Journal, 5(4), 329-330, 1963. 